\title{
Artesanía en Latinoamérica: Experiencias en el contexto de la Fabricación Digital
}

\author{
Artisanship in Latin America: Experiences in the context of Digital Fabrication
}

\author{
Pablo C. Herrera \\ Universidad Peruana de Ciencias Aplicadas, Perú \\ pablo@espaciosdigitales.org
}

\begin{abstract}
In moments when the artisanship tradition seems to disappear because of industrial production, we analyze cases where digital fabrication and visual programming were used in Latin American craft, encouraged by architects with skills in digital tools. The situations confront artisans with access to digital platforms and internet, use of learned skills, and the need to modify the technological level in their products and processes. Regional initiatives, which could change contemporary design history in the region with the establishing of a trans-disciplinary systematized synergy, show that traditional materials are used and unique components maintain their originality, from a region that attempts to enter into new global markets.
\end{abstract}

Keywords: Artisan; Latin America; Digital Fabrication; Craft.

\section{Introducción}

Según el Diccionario Oxford, artesano es un trabajador en un comercio especializado, sobre todo uno que implica hacer cosas con la mano". En el mismo contexto, el Diccionario de la Real Academia Española, define artesano como una persona que "ejercita un arte u oficio meramente mecánico". Finalmente, los artesanos de Sennett (2009) son en un sentido muy amplio, programadores informáticos, médicos o artistas, carpinteros, directores de orquesta o tejedoras (p.20). Para Sennett, "toda artesanía se funda en una habilidad desarrollada en alto grado" (p.32).

Considerando que el renacimiento de la artesanía, comprobó ser una promesa para Latinoamérica (Borges, 2015, p.14), se documentó iniciativas, patrones de implementación, origen y resultados de una selección de propuestas que relacionan artesanía y fabricación digital en su contexto regional.

Un primer objetivo permitió definir las diferencias entre el artesano en Latinoamérica y su equivalente en países que lideran el uso de tecnologías digitales emergentes. Un segundo objetivo permitió identificar en retrospectiva cómo la fabricación y programación aportan significativamente a la artesanía de la región, superando implementaciones con tecnologías previas que asociaron sólo representación y visualización. El tercer objetivo, permitió identificar la perspectiva de una línea de investigación futura, al documentar quienes transformaron el significado del objeto artesanal y lo llevaron a un sistema cultural diferente, tomando tradiciones, pero usando y adaptando materiales, integrándolas a procesos de fabricación digital, una tendencia regional que se potencia por el enorme potencial que ofrecen estas tecnologías para reproducir y modificar diseños.

\section{La artesanía en la sociedad global del siglo XXI}

La artesanía asociada a lo digital, como agente de cambio en la sociedad del siglo XXI, se estudió sistemáticamente en las conferencias de Making Futures desde el 2009 (Ferris, 2009, p.i). Allí se discutió una tendencia definida por dos tipos de usuarios. Ferris (2013) describe al primer tipo como especializado e integrado directa o indirectamente al maker movement. El segundo tipo, es el no especializado, que profesionalizó su práctica en una historia de proyectos "hágalo usted mismo" (Do it yourself, DIY), como "activistas feministas del artesanado, abogados de la simpleza material y aliados locales a favor del reciclaje y el reúso. Todos entusiasmados por iniciativas que plantean alternativas más amplias como el movimiento de transición, la agricultura urbana, los mercados locales y las ferias artesanales, e intentan llegar a nuevas audiencias a través de sitios web, blogs, grupos de interés de Facebook y YouTube". (p.5). A pesar de más de cinco años de diferencia desde el inicio de estas discusiones, aún no se puede generalizar estos tipos de usuarios a Latinoamérica, por cuanto el maker movement no es una actividad frecuente (sólo tres países realizaron una mini maker faire en nuestra región: Chile en el 2013, Colombia en el 2015 y Argentina en el 2016). Así mismo, las limitaciones tecnológicas de acceso referidas en este estudio, son la principal acción que restringió encontrar casos del segundo tipo de usuario.

Desde una perspectiva Europea, la inclinación artesanal hacia lo digital, se explica porque sus procesos de producción se movieron hacia la fabricación (Feringa, 2012:384). Tan igual como sucedió con la implementación de la programación en arquitectura, primero lo fue desde la fabricación y luego desde el diseño. Situación contraria en la región, porque los costos de equipos, hicieron que el diseño fuera el principal privilegiado y luego con los años lo sea la fabricación.

En arquitectura y diseño se discutieron casos ejemplares en diferentes conferencias como ACADIA (Feringa, 2012; 
Weston, 2013; Tang and Klimesh, 2014), CAADRIA (Senske, 2014), ICDHS (Böröcz, 2014; Kipöz and Himam, 2014) y SIGraDi (Celento, 2009; Cheng and Hegre, 2009; Anderson and Tang, 2009; Choma, 2010; Naboni and Breseghello, 2015). También Bunell $(1998,2004)$ documentó el uso de tecnologías CAD-CAM en cerámica con experiencias realizadas en tesis de doctorado y en trabajos académicos afiliados a universidades europeas. Bunell identificó programas CAD y equipos como CNC, Laser e impresoras 3D utilizados en cerámica, textil y joyería pero sin referencias al contexto latinoamericano. Aunque estos aportes son significativos en su modelo de implementación, no fue posible encontrar referencias similares al caso específico de Latinoamérica con excepción del trabajo de Borges (2011, 2015), razón por la cual, se decidió pasar a la definición y luego identificación de la muestra a trabajar.

\section{Artesano y artesanía en Latinoamérica}

Borges (2015) sostiene que en el norte del planeta, la artesanía la practican personas instruidas que buscan su autoexpresión. Al contrario en Latinoamérica, la artesanía no sólo es una actividad difundida en el campo, sino en la periferia y en barrios informales de sus pobladas ciudades como las favelas, donde colectivamente, los artesanos se enfrentan a múltiples condiciones adversas (p.11). Esto dificulta de sobre manera una implementación a corto plazo En ese sentido, José Sabogal sostuvo que "artesano es el que dispone libremente de su fuerza de trabajo y no el que trabaja para un patrón o por encargo de un contratista" (Rengifo, 1989, p.88), refiriéndose a aquellos reclutados en las zonas más deprimidas del país, en las barriadas que circulan Lima y en otras capitales sudamericanas para un pequeño empresario que tiene una fábrica y produce algo que se sindica como artesanía (Sabogal, 1974). También para Sabogal (1982), "artesano es el que esencialmente crea y recrea en la elaboración de su obra y no el que simplemente produce." (Rengifo, 1989, p.89).

Las tradiciones populares definieron a la artesanía en Latinoamérica como una "actividad económica y cultural destinada a la elaboración y producción de bienes, totalmente a mano o con ayuda de herramientas manuales, e incluso medios mecánicos (...), empleando materias primas originarias de las zonas de origen y relacionadas con un lugar de producción." (OEA, 1973). Una definición que se utiliza tardíamente en diferentes países desde sus legislaciones. En Perú por la Ley 29073 (DOP, 2007:349898) a través de su Ministerio de Comercio Exterior y Turismo, en Chile por el Concejo Nacional de la Cultura y las Artes (CNCA, 2010) y en Brasil por la Ley 13.180 a través de la Presidencia de la República (2015), reconociendo al artesano como constructor de identidad y tradición cultural y creando los primero registros nacionales para artesanos. Esta definición, no especifica la incorporación de tecnologías diferentes a la manual o mecanizada, que es una habilidad que se integra al producirse una alianza con los arquitectos.

Un caso excepcional para la región y su relación con las tecnologías digitales fue Artesanías de Colombia S.A. Creada por el Ministerio de Comercio, Industria y Turismo en 1964
(Duque, Sethi, Vencatachellum, 2005, p.70), recién con la Ley 36 (1984) se define oficialmente la profesión del artesano. Hacia finales de la década de 1990, integró por primera vez el trabajo de diseñadores y artesanos en proyectos como el Laboratorio de Diseño para innovar y mejorar la investigación y desarrollo de productos con tecnología, así como impulsar integralmente el talento humano, promoviendo la "metodología asistida por computadora, fundamentada por la interacción horizontal entre artesano y diseñador, que permitió incentivar los procesos de creatividad del artesano, visualizar y ubicar en el contexto, las alternativas que ofrece el producto al momento de la práctica, facilitando así los mecanismos de creación y recreación de su imaginario cultural.." (Duque et. al., 2005, p.73). Un modelo similar, fue adaptado en algunos países pero de manera autogestionaria y no como una política nacional. Un proceso dirigido, que sólo automatizó procesos de representación sobre otros como la fabricación que se analizan en esta investigación.

\section{Objeto artesanal}

Borges (2015) sostiene que la uniformidad y estandarización de un producto industrial es diferente al de la artesanía, porque esta última nunca es idéntica (p.14). Castrillón (1977) sostiene que "una pieza popular, aunque se trabaje en serie, nunca resulta igual a las restantes" (...) es el "resultado de una serie de adiciones que, con el tiempo, ha ido aportando la comunidad." (p. 19). En ese sentido, la programación facilita modificar las posibilidades de forma y construcción de los objetos cuando se usa código en el proceso. Así, la fabricación digital refuerza esa producción a un costo menor, porque "el costo de una parte o componente está basado en el tiempo de una máquina, no en la forma o variedad de sus partes, por lo tanto no hay cargo por la complejidad o diferencia" (SHoP, 2012, p.251). Dejando abierta la posibilidad no sólo para muchos artesanos, arquitectos y diseñadores, sino para una variedad muy amplia de objetos.

Otra situación a superar, con los objetos comprados por quienes prefieren recuerdo baratos escogidos por su valor de uso o por intelectuales, profesionales y turistas (Vargas, 1999, p.303). Rengifo (1989, p.94) destaca la posición de Sabogal (1974) respecto a la demanda de los países "metropolitanos" que desvirtúa el verdadero origen del objeto artesanal. Canclini (2004) refuerza que "los objetos artesanales producidos por grupos indígenas o campesinos, circulan por la sociedad y son apropiados por sectores urbanos, turistas, blancos y no indígenas." (p.34).

\section{Formación empírica, académica y universitaria}

Desde una visión latinoamericana, Borges (2015) sostiene que en el hemisferio norte, las técnicas artesanales se aprenden en cursos universitarios por individuos (p.11). Rengifo (1989) sostiene que el aprendizaje de la "artesanía no es escolarizado ni mediante manuales; sino que se da en el mismo proceso de socialización de los niños, tan igual como aprenden a bailar (p.91). Desde el punto de vista de quien la produce y adquiere esa habilidad, Acha (2009) sostiene que la artesanía es de formación empírica, las artes de formación académica y el diseño de formación universitaria (p.71). La fabricación digital supera estas diferencias, porque permite al 
artista o diseñador apropiarse de una tradición, para transformar la intención original de un producto único en una serie.

\section{Procedimientos metodológicos}

La investigación Latinoamericana acumulada sobre fabricación digital desde el 2004 , es el $11 \%$ del total mundial (Luli y Minto, 2015:421-433). Esta no incluye iniciativas prácticas y autogestionarias como algunas seleccionadas para esta investigación. Además, se utilizó un estudio previo realizado en sólo dos países (Herrera, 2016), y se analizó la producción de los Fab Academy en Latinoamérica establecidos en el 2009 (Latinoamérica tiene un total de 50 que representa casi el $8 \%$ del total mundial) y otros asociados al movimiento Maker. Se revisaron los resultados de tres decenas de experiencias regionales sobre fabricación digital (Sperling, Herrera, Scheeren, 2015) y se revisaron las políticas regionales y nacionales, con el fin de lograr un primer reconocimiento latinoamericano que evidencie la alianza entre artesanos, arquitectos, fabricación digital y programación. La selección de todos los casos, se presentan en esta investigación.

\section{Casos de estudio}

En un primer grupo de arquitectos, se identificó casos asociados a experiencias a) formativas, b) académicas y c) prácticas. Entre ellas se consideran resultados diversos y asociados directamente a la fabricación digital (formativa y académica) y sólo un caso que integró fabricación y programación visual (práctica). Con el objetivo de establecer una tendencia, se identificó un segundo grupo de experiencias, denominado a) experiencias no artesanales, integrado por diseñadores industriales, transformaron el uso tradicional del objeto y lado artístico.

\section{Grupo 1}

\section{a) Experiencias formativas}

Las experiencias formativas, son autogestionarias en la mayoría de casos, y están supervisadas o están dentro de un programa especializado. Tienen una mayor libertad de modelos, porque las iniciativas parten de los participantes y se potencian con el programa. Uno de ellos es el Fab Academy. (http://fabacademy.org/). Es un programa educativo que se realiza de enero a junio en 18 temas y un proyecto final. Se creó entre el MIT y el IAAC (Institud d'Arquitectura Avançada de Catalunya) para promover la fabricación digital. En el 2009 abrió su convocatoria e inició el 2010. Perú y Etiopía empezaron el proyecto, financiados por el gobierno español (Herrera y Juárez, 2013). Desde esta experiencia formativa identificamos diferentes casos, uno en Perú, otro en México y ambos relacionados a máquinas para tejer. También una iniciativa desde Costa Rica y un tercer grupo desde Colombia que descentraliza y busca en diferentes comunidades la sociabilización con la fabricación digital.

FabAcademy 2012. En el FabLab UNI, el arquitecto Walter Gonzales Arnao, fabricó un telar bajo la premisa de reducir costo y tiempo de producción. Conocido como telar fabable (fabricable en un fablab), esta máquina se produce en una hora y su costo es de alrededor de US\$100.00.

Los antecedentes se experimentaron en un curso sobre telares en la Facultad de Arquitectura de la Universidad Nacional de Ingeniería en el 2011. Los proyectos de Gonzales también fueron parte de implementaciones educativas, como es el caso del proyecto Fab Loom (Figura 1) replicado en el MediaLab Prado (Madrid, 2014). Este taller tomó las condiciones de código abierto, difundidas por su participación en el Global Fab Awards en el Fab10 realizado en Barcelona. La idea fue replicar el telar en varios FabLab de España.

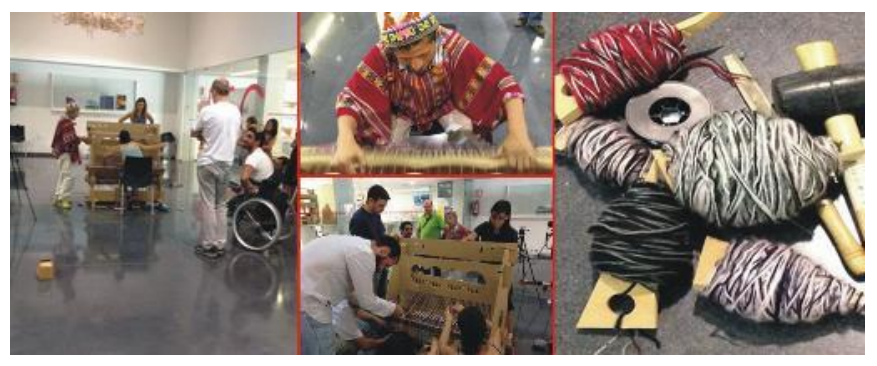

Figura 1: Actividades de replicación del telar en el MediaLab Prado de Madrid

El taller dirigido por Gonzáles en España, se centró en tres de sus diseños (Figura 2): a) el minitelar (fabricado con acrílico y cortado con láser), b) el telar de cintura y c) el telar de pedal (9 piezas de cartón prensado unidas a presión y cortadas en una CNC, optimizando hasta en un $60 \%$, usa varios materiales y contiene alrededor de 30 piezas).

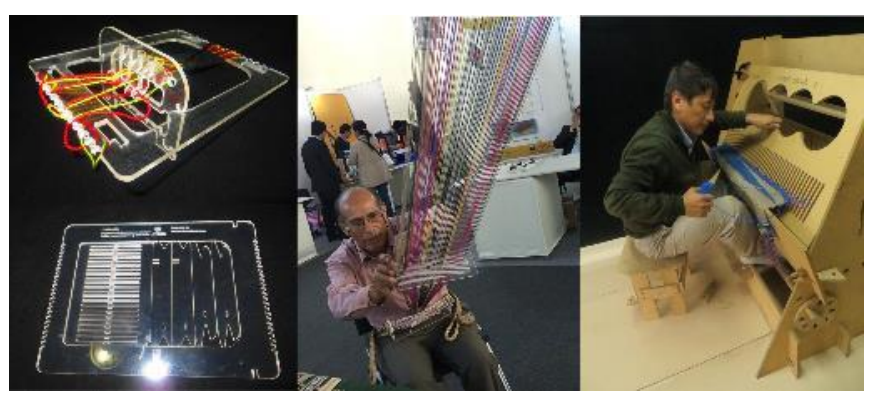

Figura 2: Propuestas del arquitecto Gonzales. Izquierda: Mini telar. Centro: Artesano Oscar Salome usando el telar andino de cinturón. Derecha: Gonzales usando su telar de Pedal.

Gonzáles recibió el premio "Diseño para el Desarrollo" por su telar a pedal y telar manual didáctico al crear una tecnología o emprendimiento que mejora la vida de las personas en situación de vulnerabilidad o pobreza, según detallan las bases de la 4aㅡ Bienal Iberoamericana de Diseño realizado en Madrid. En octubre del 2015 este invento se registró en Perú como modelo de utilidad, bajo el nombre "telar de cintura andino mejorado". Las diferentes versiones del producto fueron usadas por artesanos como el maestro Oscar Salomé Rojas, reconocido como Personalidad Meritoria de la Cultura en el 2015 por su contribución e investigación al tejido con telar de cintura. 
FabAcademy 2013, Otro caso apareció en el año 2013 en el FabLab México. El arquitecto Gonzalo Pérez se interesó en la comunidad pobre e indígena de mujeres del estado de Chiapas dedica al tejido. Uno de los contratiempos que tuvo, fue el tiempo de duración del Fab Academy limitando el proyecto original de una máquina de tejer. Se ilustra este caso, como uno de los tantos que tienen una propuesta social que puede impactar en la sociedad, pero la transición a la realidad es compleja, por los diferentes problemas técnicos a los que se enfrenta el participante en estos talleres. No ser especialistas en el problema elegido y añadir el aprendizaje de nuevas técnicas de producción, da resultados parciales o no se logran completar los objetivos iniciales. Un caso diferente al de Gonzales, que tenía experiencia en el problema y la fabricación potenció una solución específica. En el mejor de los casos, los resultados llevan a entender la naturaleza del proceso, pero con limitaciones en su resultado.

Artesanías Digitales Red FabLat. Esta red fue creada en el Séptimo congreso mundial de los FabLab (2011) e inicia actividades públicas con la red en el 2013. Su iniciativa relacionada a la artesanía se consolida en la reunión de la Red de Laboratorios de Fabricación Digital de Latinoamérica en el 2016. Sus iniciativas incluyeron talleres de diseño de empaques, así como una difusión de apoyo directo a los artesanos. Es un proyecto que recién inicia y se espera contar con resultados a corto plazo.

\section{b) Experiencias académicas}

Aconcagua FabLab. (http://www.aconcaguafablab.cl/). Es un laboratorio móvil de fabricación digital desarrollado por la escuela de arquitectura de la Pontificia Universidad Católica de Valparaíso. Se ubica en espacios públicos y se dirige a micro y pequeños empresarios del área de la artesanía, oficios tradicionales, diseño y arquitectura. Facilitó la actualización tecnológica en procesos y productos locales a 12 comunidades de la región de Valparaíso (Chile). Es una iniciativa financiada por el Fondo de Innovación para la Competitividad del Gobierno Regional de Valparaíso entre el 2014 y 2015. (Figura 3)

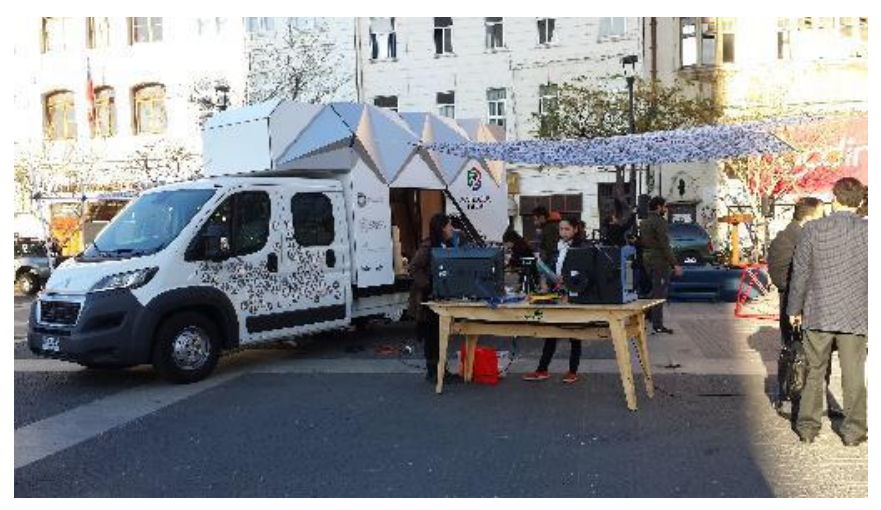

Figura 3. Unidad Móvil del FABLab Aconcagua (2015)

\section{c) Experiencias prácticas}

Desde Europa, las impresoras 3D hechas por uno mismo se usan en cerámica desde hace algunos años. Ese es el caso de Jonathan Keep que adaptó una impresora Delta y afirma que "la forma está en el código". Keep sostiene que "la computación ofrece modos muy nuevos de generar formas con cerámica, y, con el tiempo, esto 'nuevo' se volverá parte de la tradición. La alfarería siempre ha reflejado la tecnología de su tiempo, así es que para mí tiene todo el sentido generar mi trabajo en un código de computadora y luego realizarlo utilizando tecnología de impresión 3D." (Keep, 2016). Esta observación no es única, en Latinoamérica, se inició una exploración en años previos que se acercan más a recuperar propuestas propias, que adaptaciones.

great things to people, gt_2P (www.gt2p.com/). En Chile, este estudio de arquitectos tiene una propuesta que va más allá del código, fabricación y arte. Es una oficina de diseño paramétrico y fabricación digital fundada en el 2009 por iniciativa de Guillermo Parada, participante del primer taller de programación escrita realizado en la Universidad de Chile en el 2006 (Herrera, 2007).
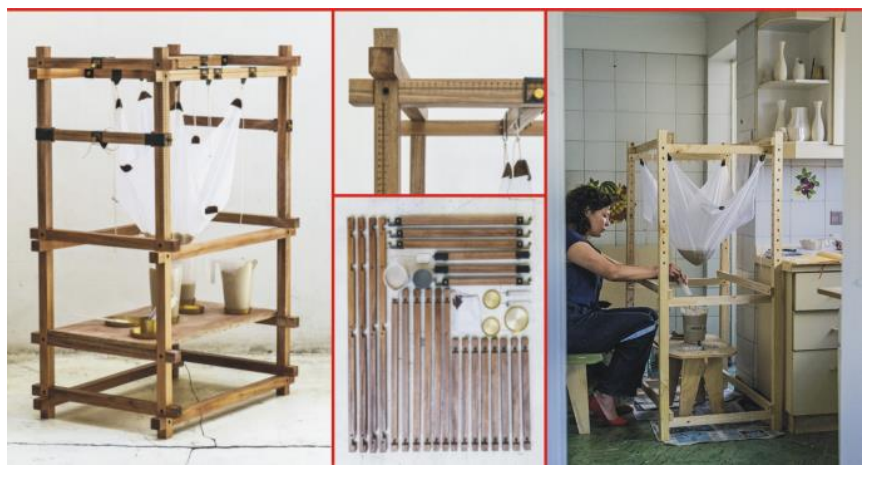

Figura 4. Catenary pottery printer por gt_2P.

Bajo la premisa, más paramétrico, menos digital, el proyecto realizado en el 2013 catenary pottery printer (Figura 4) busca experimentar el parametricismo no digital. gt_2P introduce el término digital crafting. "Esto significa mezclar tecnología digital (manejo de información, sistematización de variables, producción digital) y técnicas tradicionales (hecho a mano, industria tradicional, procedimientos y materiales locales)" (Rosso, 2014). Sus procesos integran algoritmos generativos en arquitectura, arte y diseño, lo que facilita su escalabilidad a diferentes familias de productos. Crean el ADN con reglas paramétricas que facilitan al cliente determinar el resultado de cada producto. Ellos afirman que los objetos cerámicos que producen se basan en la idea de la artesanía asistida por computador.

gt_2P explicó en el 2014 que la colección Losing my America (Figura 5) nació con el objetivo de identificar técnicas y artesanos con un alto valor sociocultural y económico, con el potencial de colaboración a través del intercambio de conocimiento en la práctica, en la búsqueda de sistematizar sus técnicas de oficio y reforzar sus economías locales." 

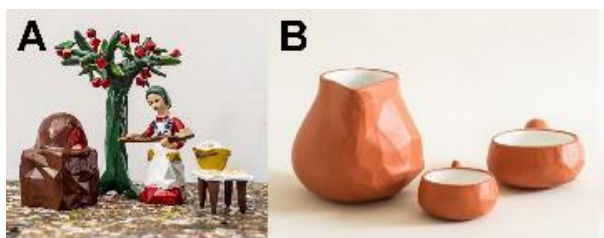

C
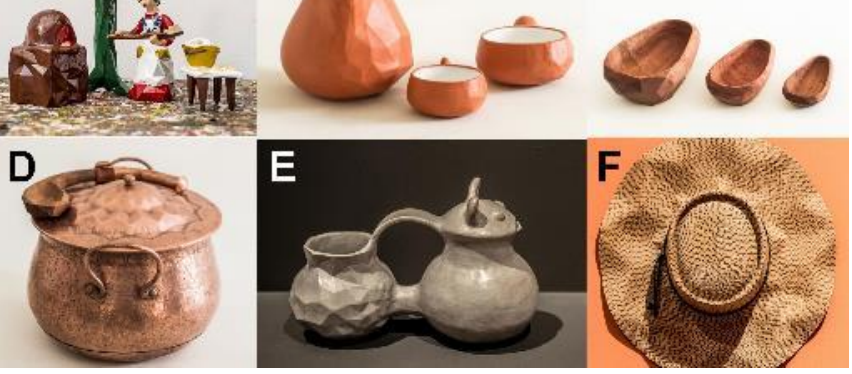

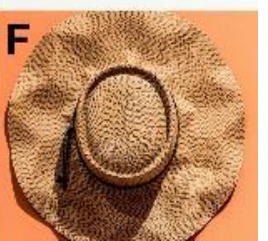

Figura 5. gt_2P con los artesanos A. Teresa Olmedo (ovenera). B. Vicson (cerámica). C. Néstor Miranda (madera). D. Juan Orellana (olla de cobre). E. Manuel González (guaco). F. Juanita Muñoz (chupalla).

Arquitectos ahora desligados a gt_2P continúan implementando el Digital Crafting, como el caso de Juan Pablo Ugarte que continuó su maestría en la GSD de Harvard, "quien explora cómo la creatividad del artesano y su lógica productiva, puede ayudar a producir flujos de trabajo en el diseño y fabricación digital, donde el dominio del material informa al diseño más activamente".

\section{Grupo 2}

\section{a) Experiencias no artesanales}

Canclini (2004) sostiene que los procesos sociales no son algo que aparece de la misma manera, así "un mismo objeto puede transformarse a través de sus usos y reapropiaciones sociales. (...) ene se sentido es etnocéntrico pensar que se ha degradado el sentido de la artesanía" (p.34). Partiendo de las definiciones regionales, se detectaron experiencias de adaptación de objetos artesanales. En ellos, no sólo varió el material, sino la forma original de los objetos, proceso y morfología, sólo permitido porque la fabricación digital permite una producción mínima, precisa y modificable. En estos casos, la intervención fue del diseñador industrial con el objeto y no con el artesano.

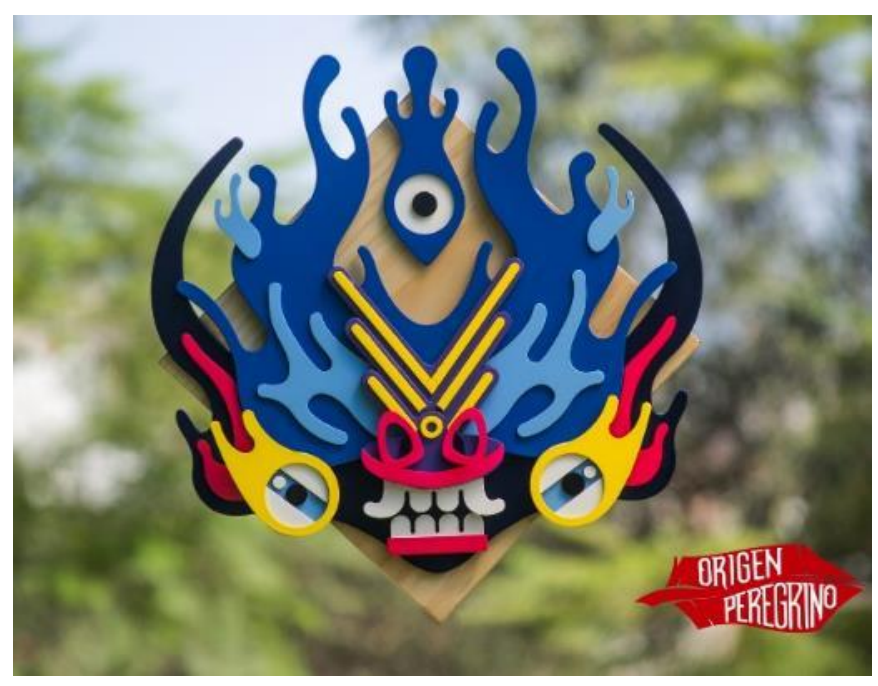

Figura 6. Adaptación de máscara por el estudio Origen Peregrino

Orígen Peregrino (http://www.origenperegrino.com), es un estudio de diseño peruano que une el trabajo de la agencia TAG Estudio Gráfico y el diseñador industrial Lau Toyosato. Ambos desarrollan piezas que a su modo de decir, "mantienen la esencia y el significado de su origen" pero realmente lo que demuestran es una pieza artística. En la Figura 6 se muestra la adaptación de una máscara tradicional utilizada en la Danza de los Diablos o Diablada en Bolivia y Perú.

Victor Freundt. Diseñador industrial peruano que recibió la primera capacitación en el FabAcademy del laaC (2009) como parte del proyecto de implementación de la red de FabLab en Latinoamérica. En el 2010, obtuvo el primer premio en el concurso Allpa Perú de Innovación en el diseño artesanal. Su producto toma como referencia una pieza de cerámica originaria del departamento peruano de Puno llamada Torito de Pucará. Utilizando la analogía del rompecabezas, a través de la técnica de encaje a presión, muy común con el trabajo de CNC logra sintetizar en pocas piezas un objeto que sirve además de baúl.

En ambos casos, aunque el origen es artesanal, los resultados son transformados para otra realidad y uso, en su conjunto sus impulsores, la describen como artesanía tradicional "con un toque muy contemporáneo".

\section{Conclusiones}

Los problemas más resaltantes de implementación, son transversales en la región, situación advertida por cada país. Especialmente porque "existe una dificultad del sector para acceder a plataformas digitales," (CNCA, 2010, p.36); "el nivel tecnológico aplicado a productos y procesos es limitado, hay resistencia a la capacitación, a aplicar lo aprendido y a modificar sus trabajos, con dificultad de acceso a Internet." (DIRCETUR Puno, 2013, p.23). Esto exige la implementación de plataformas colaborativas y redes no solo de trabajo, sino de aprendizaje y sociabilización para encaminar mejor una colaboración sostenible.

Las comparaciones del significado de artesano y artesanía en Latinoamérica, permite clarificar, que existe una diferencia marcada entre potenciar la tradición artesanal y usarla como referencia o analogía. El trabajo de arquitectos (Grupo 1) se enfocó en producir realmente una alianza y trabajo colaborativo intenso, contrario a los casos producidos por diseñadores industriales (Grupo 2) quienes adaptaron las tradiciones a sus propias necesidades.

El diseño industrial tuvo la oportunidad de ser el primer vínculo de integración con la artesanía dada su cercanía al diseño del objeto y escala, pero fue la arquitectura que demostró una integración al revitalizar el trabajo de los artesanos. Este impulso desde la arquitectura tiene sus orígenes en el retorno de estudiantes de maestría o doctorado a sus países de origen o por la capacitación recibida desde académicos externos, que evidencia ahora resultados significativos. Esto grupos de arquitectos tuvieron oportunidad de experimentar con la fabricación (Herrera, 2013) permitiendo adquirir habilidades 
que aplicaron en un contexto académico y práctico en colaboración con artesanos.

En los casos de estudio (Grupo 1), la fabricación digital permitió producir máquinas más económicas, precisas y con menos piezas con la posibilidad de reemplazar sus partes, y a futuro personalizar sus dimensiones según los usuarios (caso peruano) como se puede comparar en la Figura 7.
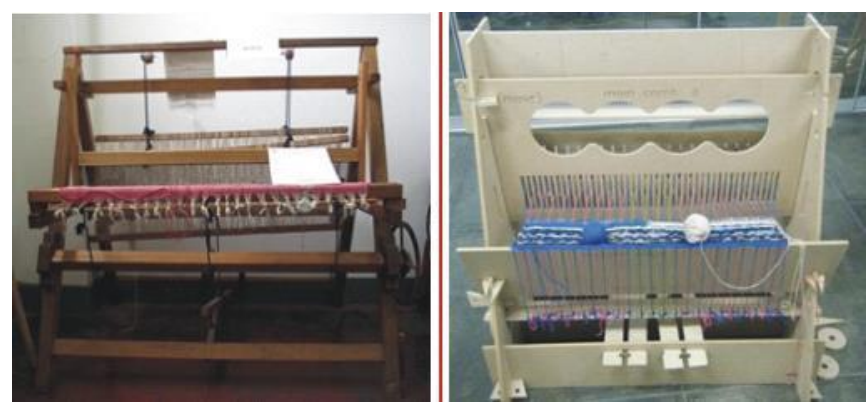

Figura 7. Comparación del telar tradicional y el fabricado por Gonzales en el FabAcademy 2012

La posibilidad de trasladar máquinas para acercar las tecnologías a poblaciones donde la llegada de centros de capacitación es limitada como la propuesta de Valparaíso, abre oportunidades de replicación no sólo local, sino para la implementación de un modelo para la región.

Los casos de estudio no serán modificables, si la memoria se queda en el objeto. gt_2P demostró que la programación flexibiliza no sólo los cambios, sino todo el proceso hasta el objeto final. La programación guiada por especialistas, que es común a los arquitectos familiarizados con Rhinoscript, Python, Grasshopper o Dynamo permite guardar el proceso de la forma del objeto, preservando la tradición de la representación y su futura modificación (caso chileno).

Esta investigación revela que se mantuvo el uso de materiales tradicionales, las piezas seguían siendo únicas y, al mismo tiempo, personalizadas por los usuarios finales. Por un lado, la fabricación digital se utilizó para automatizar procesos y mantener el carácter tradicional del objeto artesanal y por otro, se mejoraron técnicas que usaban los arquitectos y ahora implementan para sus propios procesos. Ha sido un laboratorio que permite entender desde el objeto apropiarse de una tradición que pueden llevarse en un futuro a escalas arquitectónicas, una visión necesaria que permitiría equilibrar tecnología y calidad de vida.

Estas experiencias podrían cambiar la historia del diseño contemporáneo de la región e impulsar al mismo tiempo tradiciones locales, debido a la posibilidad del control tanto del objeto como del proceso y fabricación. Se encontró que arquitectos y artesanos revitalizaron una identidad y tradición cultural sin perder el origen de sus propuestas. Esto puede impulsar las posibilidades de acceso del sector artesanal que en su mayoría se niega a traspasar los límites de sus herramientas tradicionales.
Los casos de estudio, evidencian modelos iniciales que pueden migrar a otros contextos, en especial a poblaciones emergentes, fortaleciendo raíces de identidad y cultura, que se perdían con la urbanización y migración a las ciudades por grupos de artesanos.

Considerando que Latinoamérica experimentó usar tecnologías emergentes desde la academia y no desde la práctica como sucedió en el norte del planeta. (Leach and Yuan, 2012, p.9), se concluye que el efecto de implementar programación y fabricación digital desde la década pasada en escuelas de arquitectura latinoamericanas (y muy poco en escuelas de diseño) permitió que las iniciativas de estudiantes (hoy profesionales) crezcan con una orientación por mejorar la calidad de vida de otras personas usando tecnologías a un costo prohibitivo de años previos. Esto redujo la inversión en nuevos equipos donde no sólo se favorece al artesano sino al mercado.

Estas conclusiones, permitirán reflexionar y visionar la creación de líneas de trabajo en plataformas colectivas en el contexto del Crowdthinking, donde la alianza entre arquitectos, diseñadores y artesanos otorgaría un mayor valor a la artesanía local, tanto en el objeto mismo, como es su difusión y comercialización. Sirviendo al mismo tiempo, como un modelo de aprendizaje en el que la tecnología puede y debe potenciar nuestras tradicionales.

\section{Agradecimientos}

Al concurso de Incentivo a la Investigación 2016 de la Universidad Peruana de Ciencias Aplicadas por el financiamiento de esta investigación.

\section{Referencias}

Acha, J. (2009) Introducción a la teoría de los diseños. México DF: Editorial Trillas.

Anderson, J. and Tang, M. (2014) Crafting Soft Geometry. Form and Materials informing Analog and Digital Craft Processes, 18th SIGraDi, 183-186. Montevideo.

Borges, A. (2011) Design + Craft. The Brazilian Path, Sao Paulo: Editora Terceiro Nome.

Borges, A. (2015) Craft revitalization as a change agent in Latin America. Making Futures Journal, 3, 11-14. Recuperado de http://www.plymouthart.ac.uk/documents/Adelia_Borges_KKeynote.pdf.

Böröcz, Z. (2014) Design, Crafts and Architecture in Flanders. Do They Relate in Education as in Practice? 9th ICDHS, 121-126. Aveiro, Portugal.

Bunnell, K. (2004) Craft and Digital Technology, World Craft Council 40th Annual Conference, Metsovo, Greece.

Bunnell, K. (1998) The Integration of New Technology into Ceramic Designer-Maker Practice, PhD Thesis published on CDROM, The Robert Gordon University, Aberdeen

Canclini, N. (2004) Diferentes, desiguales y desconectados. Mapas de la interculturalidad. Barcelona: Editorial Gedisa.

Cardoso, D. (2009) Generative Craft. A brief critical inquiry into design automation and design automata, 13th SIGraDi, 288-290. Sao Paulo. 
Castrillon, A. (1977) ¿Arte Popular o Artesanía? Historia y Cultura 10. Revista del Museo Nacional de Historia, 15-21. Lima.

Celento, D. (2009) Digital Craft Meets the Ancient Art of Ceramics: Would the Bauhaus Approve? 13th SIGraDi, 104-106. Sao Paulo.

Cheng, N. and Hegre, E. (2009) Serendipity and Discovery in a Machine Age: Craft and a CNC Router, ACADIA 09: reForm() Building a Better Tomorrow, 284-286. Illinois, Chicago.

Choma, J. (2010) Contested Boundaries: Digital Fabrication + Hand Craft, 14th SIGraDi, 146-149. Bogotá.

CNCA Concejo Nacional de la Cultura y las Artes. (2010) Política de fomento para la artesanía, 2010-2015, Valparaíso, Chile.

DIRCETUR Dirección Regional de Comercio Exterior y Turismo Puno (2013) Plan operativo institucional.

DOP, Diario Oficial el Peruano, (2007) Ley 29073 del artesano y del desarrollo de la actividad artesanal, Lima, Perú.

Duque, C., Sethi, R. and Vencatachellum, I. (2005) Encuentro en Diseñadores y Artesanos. New Delhi, India: Craft Revival Trust.

Feringa, J. (2012) Implicit Fabrication, Fabrication beyond Craft, The potential of Turing completeness in construction. Synthetic Digital Ecologies: ACADIA 32, 383-390. San Francisco: California College of the Arts. Recuperado de http://papers.cumincad.org/data/works/att/acadia12_383.content. pdf

Ferris, M. (2009) The crafts in the context of emerging global sustainability agendas, Making Futures Journal, vol. 1. Recuperado from http://mfarchive.plymouthart.ac.uk/journalvol1/papers/Making\%2 OFutures\%20Paper\%20Editor\%20Introduction.pdf

Ferris, M. (2013) The returns of crafting a Post-Global in the context of emerging global sustainability agendas, Making Future Journal, 3. Recuperado de http://mfarchive.plymouthart.ac.uk/journalvol3/assets/making_fut ures_editors_introduction_by_malcolm_ferris.pdf

gt_2P. Great things to People (2014). Losing my America. Recuperado de http://www.gt2p.com/Losing-my-America-1

GSD Harvard (n.d.). Doctoral Programs. Student Profiles. Recuperado de http://www.gsd.harvard.edu/\#/academic-programs/doctoralprograms/ddes/student-profiles.html

Herrera, P. (2007) Solución de problemas relacionados al diseño de superficies complejas: Experiencia de programación en la educación del arquitecto, 11th SIGraDi, 97-101. México DF.

Herrera, P. (2016) Digital Fabrication and Revival Craft in Latin America, 10th ICDHS 2016, Taipei, China.

Herrera, P. and Juarez, B. (2013) Fabrication Laboratories: Problems and possibilities of implementation in Latin America, Fab9 Research. Yokohama, Japón.
Keep, J. (2016) The Form is in the Code, Ceramics and New Technologies Symposium. Recuperado de http://www.holburne.org/planning-your-visit/whatson/talks/ceramics-symposium-abstract/

Kipöz, Ş; Himam, F (2014) Re-Inventing Traditional Textiles for the Contemporary Design Culture, 9th ICDHS, 439-444. Aveiro, Portugal.

Leach, N. and Yuan, P. (2012) Scripting the Future, Shanghai: Tongji University Press.

Luli, E. and Minto, M. (2015) Digital Fabrication in Brazil. Academic production in the last decade. In Celani, Sperling, Franco (Eds.) 16th International Conference CAAD Futures 2015, 421-433. São Paulo. Recuperado de http://papers.cumincad.org/data/works/att/cf2015_421.content.pd f

Naboni, R.; Breseghllo, L. (2015) Weaving Enclosure. Material computation and novel forms of Crafting, 19th SIGraDi, 384-391. Florianopolis, Brasil.

OEA. Organización de Estados Americanos, (1973) Carta Interamericana de las Artesanías y las Artes Populares, Washington D.C.

Rengifo, A. (1989) La Artesanía en la obra de José Sabogal Wiesse. Lima: Centro de Proyectos Integrales en Base a la Alpaca.

Rosso, A. (n.d.) Digital Crafting with gt2P Chilean Collective. Recuperado de https://artemest.com/magazine/new-vision/gt2pdigital-crafting

Sabogal, J. (1974) Artesano y Artesanías. Diario El Comercio. Lima, 13 de noviembre de 1974.

Sabogal, J. (1982) La cerámica de Piura. Quito, Ecuador: Instituto Andino de Artes Populares.

Sennett, R. (2009) El artesano. Barcelona: Editorial Anagrama.

Senske, N. (2014) Digital Minds, Materials, and Ethics: Linking Computational Thinking and Digital Craft, 19th CAADRIA, 841850. Hong Kong

SHoP (2012) SHoP Out of Practice, New York: Monacelli Press.

Sperling, D.; Herrera, P.; Scheeren, R. (2015) Migratory Movements of Homo Faber: Mapping Fab Labs in Latin America, CAAD Futures 2015, 405-421. Sao Paulo, Brasil.

Vargas, G. (1999) Flexible Looms: Weavers' Organizations In Chiapas, México, Urban Anthropology and Studies of Cultural Systems and World Economic Development, 28, 299-325.

Weston, M. (2013) CNC Sponge-Forming and Parametric Slip Casting: Experiments in the hybridization of computation and traditional craft for architectural ceramics, ACADIA 13: Adaptive Architecture, 455-456. Cambridge. 